\title{
Prática da enfermagem na conservação de vacinas*
}

\author{
Nursing practice in vaccines preservation
}

Práctica de enfermería en la conservación de vacunas

Valéria Conceição de Oliveira ${ }^{1}$, Eliete Albano de A. Guimarães ${ }^{2}$,
Inês Alcione Guimarães ${ }^{3}$, Letícia Helena Januário $^{4}$, Ione Carvalho Ponto ${ }^{5}$

\section{RESUMO}

Objetivo: Identificar o conhecimento e o cumprimento das recomendações técnicas do Programa Nacional de Imunização sobre a conservação dos imunobiológicos nas Unidades Básicas de Saúde de um município da região Centro-Oeste de Minas Gerais. Metodos: Estudo descritivo transversal realizado em 26s Unidades Básicas de Saúde abrangendo 21 enfermeiros e 49 avaliadores de enfermagem entrevistados. Resultados: Foram observadas algumas deficiências que podem interferir na eficácia do Programa Nacional de Imunização, tais como, manutenção da rede de frio inadequada, desconhecimento da conduta técnica de limpeza quinzenal, desconhecimento das vacinas que podem sofrer congelamento sem risco de inativação e falta de ambientação da bobina de gelo reciclável. Conclusão: Foram sugeridos investimentos na formação, informação e capacitação dos profissionais responsáveis pela prática de conservação dos imunobiológicos.

Descritores: Refrigeração; Programas de imunização; Vacinas.

\begin{abstract}
Objective: To identify the knowledge and compliance with the technical recommendations of the Brazilian National Immunization Program regarding immunobiological preservation. Methods: A cross-sectional descriptive study was conducted among 21 registered nurses and 49 licensed practical nurses from twenty-six primary health care units in a municipal district in the State of Minas Gerais, Brazil. Results: Several deficiencies that can affect the effectiveness of the recommendations of the National Immunization Program were identified: inadequate management of temperature of the refrigerator, lack of knowledge of the technical procedure for biweekly cleaning of the refrigerator, and lack of awareness of the recyclable ice box. In addition, there was a lack of knowledge of vaccines that would continue active even though they had been frozen. Conclusion: There is need for education and training of professionals responsible for the immunobiological preservation.
\end{abstract}

Keywords: Refrigeration; Immunization programs; Vaccines.

\section{RESUMEN}

Objetivo: Identificar el conocimiento y el cumplimiento de las recomendaciones técnicas del Programa Nacional de Inmunización sobre la conservación de los inmunobiológicos en las Unidades Básicas de Salud de un municipio, en la región Centro Oeste de Minas Gerais. Métodos: Estudio descriptivo transversal realizado en 26 Unidades Básicas de Salud donde fueron entrevistados 21 enfermeros y 49 auxiliares de enfermería. Resultados: Fueron observadas algunas deficiencias que pueden interferir en la eficacia del Programa Nacional de Inmunización, tales como, manutención del circuito de frío inadecuada, desconocimiento de la conducta técnica de limpieza quincenal, desconocimiento de las vacunas que pueden sufrir congelamiento sin riesgo de inactivación, y, falta de ambientación de la bobina de hielo reciclable. Conclusión: Fue sugerido realizar inversiones en la formación, información y capacitación de los profesionales responsables por la práctica de conservación de los inmunobiológicos.

Descriptores: Refrigeración; Programas de inmunización; Vacunas.

\footnotetext{
"Estudo desenvolvido nas unidades básicas de saúde de um Município da Região Centro-Oeste de Minas Gerais (MG), Brasil.

${ }_{1}^{1}$ Mestre em Enfermagem. Professora da Universidade Federal de São João del Rei (MG), Brasil.

${ }^{2}$ Pós-graduanda (Doutorado) em Ciências da Saúde CPqRR/FIOCRUZ. Professora da Universidade Federal de São João del Rei (MG), Brasil.

${ }^{3}$ Pós-graduanda (Mestrado) da Universidade do Estado de Minas Gerias / Fundação Educacional de Divinópolis (UEMG/FUNEDI). Gerente de Unidade Básica da Secretaria Municipal de Saúde Divinópolis (MG), Brasil.

${ }^{4}$ Pós-graduanda (Doutorado) em Saúde da Criança e do Adolescente da Universidade de São Paulo - USP - Ribeirão Preto (SP), Brasil. Professor auxiliar do Centro de Ciências da Saúde da Universidade Federal de São João del Rei (MG), Brasil.

' Livre-docente. Professora da Escola de Enfermagem de Ribeirão Preto da Universidade de São Paulo - USP - Ribeirão Preto (SP), Brasil.
} 


\section{INTRODUÇÃO}

A imunização representa uma das medidas mais efetivas na prevenção de doenças, principalmente na faixa etária de zero a cinco anos de idade, por reduzir a morbimortalidade por doenças imunopreviníveis ${ }^{(1)}$. Muitas das doenças que assolavam o país nas três últimas décadas foram erradicadas ou estão sob controle, tais como a poliomielite, o sarampo, a varíola e a raiva humana, a partir da adoção destas medidas de imunização específica.

O Ministério da Saúde, através do Programa Nacional de Imunização (PNI), criado na primeira metade da década de 1970, vem, desde então, contribuindo significativamente para esta mudança do perfil epidemiológico do país. O PNI tem a função de manter sob controle as doenças imunopreviníveis no Brasil, definindo prioridades para a imunização e provendo os Estados e municípios com estes imunobiológicos ${ }^{(2)}$. Também cabe ao PNI orientar as condutas adequadas à conservação, manipulação, transporte e aplicação dos imunobiológicos.

Os imunobiológicos são produtos termolábeis que necessitam de refrigeração, a fim de manter sua capacidade imunizante. A estabilidade de uma vacina pode ser afetada também por outros fatores como a luz, a umidade e a cepa vacinal. A importância destes fatores sobre a manutenção da qualidade dos imunobiológicos é de tal maneira relevante, que sempre foi objeto de norma técnica do PNI, constituindo um manual específico: Manual de Rede de Frio ${ }^{(3)}$.

O PNI define como rede de frio o complexo de ligação entre os níveis nacional, estadual, regional, municipal e local, incluindo o armazenamento, o transporte e a manipulação dos imunobiológicos em condições adequadas. As vacinas são conservadas em temperaturas específicas, levando em conta sua composição. No nível nacional, alguns imunobiológicos são conservados em temperaturas negativas, já no nível local são refrigeradas entre $+2^{\circ} \mathrm{C} \mathrm{a}$ $+8^{\circ} \mathrm{C}$, em refrigeradores exclusivos ${ }^{(3)}$.

Nas Unidades Básicas de Saúde (UBS), a continuidade da rede de frio, ou seja, a manutenção da qualidade dos imunobiológicos, no que diz respeito à conservação e à administração dos mesmos, é atividade exclusiva da equipe de enfermagem.

Também em outros países, como na Espanha, esta prática é de competência da enfermagem. Em trabalho realizado nos Centros de Atenção Primária na cidade de Madrid, 97,7\% dos profissionais responsáveis pela rede de frio são da equipe de enfermagem ${ }^{(4)}$.

A conservação de imunobiológicos no nível local, principalmente nas UBS, apesar de incontestável relevância, apresenta falha significativa que pode colocar em risco a eficácia da imunização, não só em países em desenvolvimento, mas também nos desenvolvidos ${ }^{(5)}$.

Um estudo foi realizado em centros de Atenção Primária na cidade de Madrid, com o objetivo de avaliar como se realiza a manutenção da cadeia de frio e o grau de informação dos responsáveis por esta manutenção. Neste estudo, os pesquisadores recomendaram um reforço na informação e na formação dos responsáveis pela vacinação, quanto à estabilidade destes produtos ${ }^{(4)}$.

Também em estudos realizados no Brasil, foram descritas falhas no cumprimento das recomendações para a conservação de vacinas. $\mathrm{Na}$ cidade de Bocaiúva, interior de Minas Gerais, foi realizada uma pesquisa para avaliar a efetividade da vacina antiamarílica utilizada pelos serviços de saúde pública. Os pesquisadores observaram que a soropositividade para o vírus amarílico ficou abaixo dos níveis esperados, levando-os a concluir que este resultado pôde ser atribuído a possíveis falhas na conservação e na aplicação das vacinas ${ }^{(6)}$.

A experiência como enfermeiras de UBS, e como docentes, têm-nos evidenciado uma diversidade de condutas na conservação dos imunobiológicos nas diversas UBS, e até mesmo numa única unidade. Também percebemos ocorrências impróprias como a presença de vacinas que deveriam já ter sido desprezadas em razão do tempo de diluição, armazenadas nos refrigeradores. Esta realidade inquietou-nos e motivou a realização de um estudo para melhor dimensionamento desta divergência de condutas percebida na conservação de vacinas.

Neste sentido, este estudo objetivou identificar o conhecimento e o cumprimento das recomendações técnicas do Programa Nacional de Imunização sobre a conservação dos imunobiológicos nas Unidades Básicas de Saúde de um munićpio, na região centro oeste de Minas Gerais.

\section{MÉTODOS}

Trata-se de um estudo descritivo, transversal, realizado em município mineiro da Macrorregional Oeste. O município encontra-se a $123 \mathrm{Km}$ de Belo Horizonte, e conta com uma população de 210.000 habitantes (IBGE/2007). Atualmente, é a cidade Pólo Regional de Saúde e possui 26 UBS, sendo 11 destas, Estratégias de Saúde da Família.

O município conta com duas escolas de enfermagem de nível técnico e uma de nível superior.

Após assinatura do Termo de Consentimento pelo Gestor da Secretaria Municipal de Saúde, foram agendados encontros com os gerentes e enfermeiros responsáveis técnicos das UBS, para esclarecimentos sobre o estudo e conformidades necessárias para a participação dos sujeitos envolvidos com a Rede de Frio.

A população participante constituiu-se de 70 profissionais de enfermagem (21 enfermeiros e 49 auxiliares de enfermagem) responsáveis pela vacinação na atenção primária em saúde.

Os dados foram coletados, após assinatura do Termo de Consentimento Livre e Esclarecido, por duas pesquisadoras integrantes do projeto, que utilizaram entrevistas estruturadas e um roteiro enfocando a conservação dos imunobiológicos na prática diária da enfermagem na imunização. As pesquisadoras realizaram um teste piloto objetivando evitar divergências de entendimento, reduzindo a possibilidade 
deste viés. Os dados foram organizados e sistematizados no programa Epi-info, versão 6,0.

Este estudo foi realizado com a observância da Resolução 196/96 do Conselho Nacional de Saúde e aprovado pelo Conselho de Ética do Hospital São João de Deus (MG).

\section{RESULTADOS}

Os sujeitos da pesquisa foram 70 entrevistados, sendo 21 (30\%) enfermeiros e 49 (70\%) auxiliares de enfermagem. Esta amostra representa $91 \%$ da totalidade dos 23 enfermeiros e $64 \%$ do total de 73 auxiliares responsáveis pela imunização nas UBS do município.

A média de idade dos participantes foi de 39 anos, sendo a mínima de 22 anos e a máxima de 62 anos. O sexo predominante foi o feminino com $64(91,4 \%)$ dos participantes.

Quanto ao tempo de serviço em sala de vacina, oito entrevistados $(11,4 \%)$ tinham menos de um ano de experiência, sendo que quatro deles tinham apenas um mês

Tabela 1 - Respostas sobre inativação dos imunobiológicos por congelamento, segundo categoria profissional e tempo de serviço em sala de vacina. Divinópolis, 2005.

\begin{tabular}{|c|c|c|c|c|c|c|c|c|}
\hline \multirow{4}{*}{$\begin{array}{l}\text { Tempo de Serviço } \\
\text { (anos) }\end{array}$} & \multicolumn{8}{|c|}{ Inativação dos Imunobiológicos por Congelamento } \\
\hline & \multicolumn{4}{|c|}{ Enfermeiros } & \multicolumn{4}{|c|}{ Aux. Enf. } \\
\hline & \multicolumn{2}{|c|}{ Corretas } & \multicolumn{2}{|c|}{ Incorretas } & \multicolumn{2}{|c|}{ Corretas } & \multicolumn{2}{|c|}{ Incorretas } \\
\hline & $\mathrm{n}$ & $\%$ & $\mathbf{n}$ & $\%$ & $\mathbf{n}$ & $\%$ & $\mathbf{n}$ & $\%$ \\
\hline De $0-\mid 1$ & - & - & - & - & 1 & 14.3 & 6 & 85,7 \\
\hline De $1-\mid 2$ & 1 & 16,5 & 1 & 50,0 & - & - & 1 & - \\
\hline De 2 - 4 & - & - & 4 & - & 1 & 25,0 & 3 & 75,0 \\
\hline De 4 - 10 & 5 & 83,7 & 5 & 50,0 & 4 & 19,0 & 17 & 81,0 \\
\hline De 10 e mais & - & - & 4 & 100,0 & 6 & 37,5 & 10 & 62,5 \\
\hline Total & 6 & 28.6 & 15 & 71.4 & 12 & 24.5 & 37 & 75,5 \\
\hline
\end{tabular}

Tabela 2 - Conhecimento dos profissionais de enfermagem quanto à duração das vacinas após abertura do frasco. Divinópolis, 2005.

\begin{tabular}{lcc}
\hline Vacina & $\mathbf{n}$ & $\%$ \\
\hline Antiamarílica & 51 & 72,9 \\
Conhece & 19 & 27,1 \\
Desconhece & & \\
BCG & 43 & 61,4 \\
$\quad$ Conhece & 27 & 38,6 \\
Desconhece & & \\
DPT & 52 & 74,3 \\
Conhece & 18 & 25,7 \\
Desconhece & & \\
Dupla adulto & 62 & 88,6 \\
Conhece & 8 & 11,4 \\
Desconhece & & \\
Hepatite b & 62 & 88,6 \\
Conhece & 8 & 11,4 \\
Desconhece & & \\
Influenza & 42 & 60,9 \\
Conhece & 27 & 39,1 \\
Desconhece & & \\
Sabin & 49 & 70,0 \\
Conhece & 21 & 30,0 \\
Desconhece & & \\
Tetravalente & 42 & 60,0 \\
Conhece & 48 & 40,0 \\
Desconhece & & 67,1 \\
Triviral & 23 & 32,9 \\
Conhece & & \\
Desconhece & & \\
\hline
\end{tabular}

de trabalho; 31 participantes (44,3\%) apresentavam entre quatro e dez anos de experiência em sala de vacina. A grande maioria, 51 (72,9\%) dos participantes possuía experiência superior a quatro anos na atividade.

Verifica-se, através dos resultados, que 26 entrevistados $(37,1 \%)$ participaram de capacitação em imunização, promovida pela Gerência Regional de Saúde em parceria com a Secretaria Municipal de Saúde do município. Os demais entrevistados (62,9\%) nunca participaram de qualquer curso sobre imunização, além da formação específica requerida para o exercício da função.

Em relação à temperatura ideal para a conservação de vacinas no nível local de saúde, 59 entrevistados (84,3\%) responderam corretamente com o preconizado pelo $\mathrm{PNI}^{(2)}$.

Quanto à limpeza e o degelo do refrigerador de imunobiológicos, 51,4\% responderam que realizam a limpeza a cada 15 dias, 8,6\% não sabem ou não responderam, e $40,0 \%$ dos entrevistados responderam que realizam o procedimento de acordo com uma periodicidade que não está em consonância com orientações do PNI.

A Tabela 1 mostra o resultado da avaliação das respostas sobre as vacinas utilizadas nas UBS que podem sofrer congelamento sem risco de inativação. As respostas foram avaliadas em corretas e incorretas, conforme orienta o Manual do $\mathrm{PNI}^{(2)}$.

Foi perguntado aos entrevistados qual seria sua conduta ao se deparar com uma temperatura do refrigerador acima do nível considerado adequado para a conservação das vacinas. Observou-se que $35,7 \%$ comunicariam o aumento da temperatura à Secretaria Municipal de Saúde e 32,9\% 
responderam condutas variadas que não estão em conformidade com o PNI.

Sobre a ambientação das bobinas de gelo reciclável, 48 participantes $(68,6 \%)$ disseram não realizar a ambientação.

Observam-se na Tabela 2 informações sobre a validade das vacinas após abertura do frasco.

\section{DISCUSSÃO}

A partir da análise dos resultados deste estudo, pode-se observar algumas deficiências na manutenção da Rede de Frio nas UBS, que podem interferir na eficácia da Imunização no município.

A temperatura de conservação dos imunobiológicos no nível local deve permanecer entre $+2^{\circ} \mathrm{Ce}+8^{\circ} \mathrm{C}$. Apesar do número de respostas corretas ser significativamente alto, é preocupante que 11 entrevistados $(15,7 \%)$ não saibam a temperatura de conservação de vacinas na rede local.

A limpeza e o degelo do refrigerador também constituem uma conduta técnica importante na manutenção das condições ideais das vacinas. Conforme normas do PNI, o degelo e a limpeza interna da geladeira tipo doméstica devem ser realizados a cada 15 dias ou quando a camada de gelo atingir $0,5 \mathrm{~cm}^{(3)}$. O presente estudo revelou que $40 \%$ dos profissionais desconhecem essa norma. Em estudo realizado em Vinhedo - SP, objetivando avaliar a qualidade da estrutura e do processo de operacionalização da rede de frio, observou-se que os refrigeradores eram descongelados e limpos quinzenalmente para manter seu adequado funcionamento ${ }^{(7)}$.

Algumas vacinas podem congelar sem alteração do seu poder imunogênico como a Febre Amarela, Sabin e Triviral. Entretanto, algumas delas como a BCG, dupla adulto (dt), tríplice bacteriana (DPT), hepatite b, influenza, tetravalente, uma vez congeladas, podem perder potência comprometendo a sua proteção imunogênica ${ }^{(8)}$.

A Tabela 1 apresenta o resultado da avaliação das respostas sobre o conhecimento quanto à inativação dos imunobiológicos por congelamento. Como se pode observar, a maioria de enfermeiros e auxiliares de enfermagem não respondeu corretamente quais vacinas podem ou não ser congeladas. Entretanto, a avaliação desta questão foi feita sobre o acerto de todos os itens (nove vacinas) relacionados no instrumento de pesquisa, ou seja, foram consideradas como "corretas" as respostas do profissional, que acertou todos os itens.

Entende-se que, se os profissionais lidam diariamente com todas as vacinas citadas, necessariamente, todos devem conhecer as possibilidades de inativação das vacinas com as quais trabalham. Notou-se que parte dos entrevistados (19) relatou que nenhuma, ou que apenas uma vacina podia sofrer congelamento. Estas respostas podem ser avaliadas de forma positiva, se considerado que nenhuma vacina do PNI deve ser congelada no nível local. Alguns autores corroboram essa afirmativa, ao dizer que o baixo índice de conhecimento sobre as vacinas que podem ser congeladas não coloca em risco a efetividade do programa, pois nas UBS não utilizam freezers e não se armazenam vacinas no congelador ${ }^{(5)}$. Por outro lado, desconhecer quais imunobiológicos podem ser congelados expõe a unidade de saúde ao risco de perda desnecessária de vacinas que não sofrem inativação caso, eventualmente, venham a ser congeladas nas UBS.

O refrigerador da sala de vacina pode deixar de funcionar adequadamente, o que poderá ser verificado por meio da leitura do termômetro de máxima e mínima. Ao observar um aumento de temperatura sem motivos conhecidos, devese suspeitar do imunobiológico que está sob refrigeração. Neste caso, a norma técnica sugere as seguintes providências: suspender de imediato o uso do produto mantendo-o sob refrigeração adequada, comunicar ao responsável técnico do Nível Central de Saúde, registrar e notificar as observações no mapa de controle diário da temperatura e ao serviço de vigilância em saúde ${ }^{(3)}$. Em relação a isso, as respostas dos entrevistados foram as mais variadas possíveis. Destacam-se as seguintes:

- Nove entrevistados relataram que ajustam o termostato após a detecção de aumento da temperatura e não colocam o imunobiológico sob suspeita. A regulagem do termostato é realizada para evitar que, durante a noite, a parte posterior da primeira prateleira atinja temperaturas negativas ${ }^{(3)}$.

- Sete entrevistados descreveram várias condutas tais como: "abre a geladeira", "tira gelox", "aguarda 24 horas", "limpa a geladeira e depois retorna com as vacinas", "não faz nada". Essas afirmações nos trazem uma grande preocupação, uma vez que algumas vacinas são muito sensíveis ao aumento de temperatura, o que nem sempre é percebido pela simples inspeção, impondo certa insegurança quanto à sua efetividade. É sabido que a imunização representa uma das medidas mais eficazes na prevenção de doenças, reduzindo a morbimortalidade por doença imunoprevenível, sendo o principal meio de manutenção dessa estratégia a adequada conservação dos imunobioló-gicos ${ }^{(2,4-5)}$.

A ambientação da bobina de gelo reciclável também é um procedimento técnico de grande importância na conservação dos imunobiológicos e deve ser realizada sempre que for acondicionar vacinas em caixas térmicas nas diversas instâncias, inclusive para uso em sala de vacina. Dos participantes, $68,6 \%$ entrevistados disseram não realizar essa ambientação. Essa detecção é grave, uma vez que as bobinas de gelo, ao serem retiradas do congelador, estão com temperatura próxima de $-7^{\circ}$ C (sete graus Celsius negativos) e se forem colocadas imediatamente na caixa, com os imunobiológicos, sem controle prévio da temperatura, expõem-se estas vacinas ao risco de congelamento. $\mathrm{O}$ congelamento, como se sabe, pode inativar vacinas como a BCG, DPT e a tetravalente. A precipitação de uma vacina pode ocorrer devido à permanência da mesma a uma temperatura inferior a $0^{\circ} \mathrm{C}$ (zero graus Celsius), por período de tempo de vários dias, ou acúmulo de períodos parciais de tempo ${ }^{(3,9)}$. Este resultado traduz uma situação preocupante e relevante para a gestão, visto que os profissionais de enfermagem constituem a equipe técnica responsável pela conservação e administração dos imunobiológicos na rede pública de saúde.

As vacinas reconstituídas representam um meio ideal para 
o crescimento de microorganismos. Neste sentido, o conhecimento do tempo de validade das vacinas após reconstituição/diluição é relevante. A maioria das vacinas, após abertas ou diluídas, devem ser usadas por um período determinado de tempo, por exemplo, a antiamarílica que tem duração de quatro horas após diluída, e a BCG, seis horas após a reconstituição. Ao analisar a Tabela 2, observou-se que mais de $27 \%$ dos entrevistados responderam incorretamente sobre o tempo de duração da vacina antiamarílica, e mais de $30 \%$ responderam incorretamente sobre as vacinas BCG, influenza, Sabin, triviral. As respostas incorretas sobre a tetravalente chegaram a $40 \%$. Observa-se que o maior número de respostas corretas refere-se às vacinas sobre as quais não há limite de duração de tempo em condições adequadas de conservação e dentro do prazo de validade do fabricante como a dt, e hepatite b $(88,6 \%)$ e a DPT $(74,3 \%)$.

Considerando o procedimento de conservação de vacinas necessário e prioritário para a eficácia na redução das doenças imunopreveníveis, preocupa-nos os resultados encontrados, visto que, como mencionado, trata-se de um município pólo regional, o qual conta com escolas de enfermagem, tanto de nível técnico, quanto de nível superior.

\section{CONCLUSÕES}

É indiscutível a importância da manutenção da rede de

\section{REFERÊNCIAS}

1. Oliveira MAC, Takahashi RF. Questões práticas relacionadas à aplicação de vacinas. In: Farhat $\mathrm{CK}$, Carvalho ES, Weckx LY, Carvalho LHF, Succi RCM, editores. Imunizações: fundamentos e prática. 4a. ed. São Paulo: Atheneu; 2000. p.137-48.

2. Almeida MM. Conservação e manipulação de imunobiológicos. In: Farhat CK, Carvalho ES, Weckx LY, Carvalho LHF, Succi RCM, editores. Imunizações: fundamentos e prática. $4^{\mathrm{a}}$ ed. São Paulo: Atheneu; 2000. p.125-35.

3. Ministério da Saúde (BR). Fundação Nacional de Saúde. Manual de rede de frio. $3^{a}$ ed. Brasília: FUNASA; 2001.

4. Ortega Molina P, Astasio Arbiza P, Albaladejo Vicente R, Gómez Rábago ML, de Juanes Pardo JR, Domínguez Rojas V. Cadena del frío para la conservación de las vacunas en los centros de atención primaria de un area de Madrid: mantenimiento y nivel de conocimientos. Rev Esp Salud Publica. 2002;76(4):333-46.

5. Aranda CMSS, Moraes JC. Rede de frio para a frio para assegurar que todos os imunobiológicos mantenham suas características imunogênicas desde o laboratório produtor até a administração da vacina à comunidade.

Foram observadas deficiências na manutenção da rede de frio nas UBS do município estudado, que podem interferir na efetividade do Programa Nacional de Imunização.

A equipe de enfermagem é a responsável pela imunização na rede municipal, neste sentido, é de extrema importância maior investimento na formação acadêmica dos profissionais. Também é evidente a necessidade de aperfeiçoamento dos profissionais, uma vez que as normas de vacinação estão em constantes mudanças, e a introdução de imunobiológicos no calendário vacinal é frequente.

Desta forma, a educação permanente na formação dos profissionais responsáveis pela imunização parece-nos uma iniciativa com o potencial de mudança necessária para que a equipe de enfermagem possa assegurar a qualidade das vacinas disponibilizadas à população.

O estudo também evidenciou a necessidade de mais pesquisas sobre a prática da enfermagem na conservação dos imunobiológicos. Entendemos que a necessidade de mais estudos justifica-se, pela relevância deste procedimento para a atenção primária à saúde e por constituir-se numa atividade realizada, quase exclusivamente, pela equipe de enfermagem. Não obstante, a referência específica é escassa.

conservação de vacinas em unidades públicas do município de São Paulo: conhecimento e prática. Rev Bras Epidemiol. 2006;9(2):172-85.

6. Guerra HL, Sardinha TM, Rosa APAT, Costa MFL. Efetividade da vacina aniamarílica 17D: uma avaliação epidemiológica em serviços de saúde. Rev Panam Salud Publica. 1997;2(2):115-20.

7. Escobar EMA, Adami NP, Silva CV. Avaliação da qualidade da rede de frio do programa de imunização de Vinhedo-SP. Acta paulista Enferm. 2002;15(3):7-14.

8. Nelson CM, Wibisono H, Purwanto H, Mansyur I, Moniaga V, Widjaya A. Hepatitis B vaccine freezing in the Indonesian cold chain: evidence and solutions. Bull World Health Organ. 2004;82(2):99-105.

9. Ortega Molina P, Astasio Arbiza P, Albaladejo Vicente R, Aráosla Martínez P, Villanueva Orbáiz R, Ramón de Juanes Pardo J. Mantenimiento de la cadena del frío para las vacunas: una revisión sistemática. Gac Sanit. 2007;21(4):343-8. 